\title{
Des passions funestes et salutaires. Les histoires tragiques selon Jean-Pierre Camus
}

\section{Frank Greiner}

\section{(2) OpenEdition}

1 Journals

Édition électronique

URL : https://journals.openedition.org/studifrancesi/20587

DOI : 10.4000/studifrancesi.20587

ISSN : 2421-5856

Éditeur

Rosenberg \& Sellier

\section{Édition imprimée}

Date de publication : 1 décembre 2019

Pagination : 442-454

ISSN : 0039-2944

\section{Référence électronique}

Frank Greiner, « Des passions funestes et salutaires. Les histoires tragiques selon Jean-Pierre

Camus », Studi Francesi [En ligne], 189 (LXIII | III) | 2019, mis en ligne le 01 décembre 2020, consulté le 11 novembre 2021. URL : http://journals.openedition.org/studifrancesi/20587 ; DOI : https://doi.org/ 10.4000/studifrancesi.20587

\section{(c) (i) (9)}

Studi Francesi è distribuita con Licenza Creative Commons Attribuzione - Non commerciale - Non opere derivate 4.0 Internazionale. 


\title{
Des passions funestes et salutaires. Les histoires tragiques selon Jean-Pierre Camus
}

\begin{abstract}
The three collections of tragic stories of Jean-Pierre Camus, L'Amphithéâtre sanglant, Les Spectacles d'borreur and Les Rencontres funestes, were already the object of several studies which insist most of the time on the dissuasive role of the terrible scenes represented in the eyes of the reader with a particular sense of macabre detail. Our purpose in this article is to nuance this summary approach by taking into account his conception of the passions explained in the Traitté des passions de l'âme and the Traité de la Réformation intérieure. It seems in the light of these works that the intention of Camus in his stories is not simply to illustrate the idea that nothing escapes the vigilance of the Providential Justice and that God punishes inevitably all delinquents. Certainly Camus tries to terrify to improve, but also incites to think. Indeed the Providentialism of the bishop of Belley gives an important place to a rational conception of Justice, because it invites the readers to understand the criminal behaviours in relationship to passionate mechanisms causing their fatal development. This particular representation of the crime, that also focuses on the psychological issues, is rich with many repercussions on the narrative structure, rhetoric and morality of his tragic stories.
\end{abstract}

«Terrifier pour édifier» aurait pu être la devise des auteurs d'histoires tragiques qui au temps de la Contre-Réforme mirent leur plume au service d'une «pastorale de la peur» ${ }^{1}$ avec le dessein de combattre l'immoralité, l'impiété et l'hérésie. La stratégie de la dissuasion entra-t-elle aussi dans le projet littéraire du célèbre disciple de François de Sales, Jean-Pierre Camus, et, si tel était probablement le cas, comment le sentiment de la crainte était-il utilisé par lui? Il est d'abord frappant de constater que dans son œuvre d'écrivain les histoires tragiques se réduisent à la portion congrue: trois recueils parmi les vingt-et-un que compte son abondante production de nouvelliste: L'Amphithéâtre sanglant, Les Spectacles d'borreur (1630) et Les Rencontres funestes ${ }^{2}$ (1644) qui seuls seront étudiés dans ces quelques pages du fait de l'homogénéité de leur inspiration et de leur situation singulière parmi les textes camusiens. On retrouve dans ces trois ouvrages les principales caractéristiques du genre tel qu'il était pratiqué avant lui: les histoires tragiques de Camus, comme celles de François de Belleforest et de François de Rosset dont il se réclame ${ }^{3}$, sont des récits brefs donnés comme des témoignages réalistes concernant des événements sinistres en tous genres (drames passionnels, affaires criminelles

(1) Selon l'expression de J. Delumeau, Le Péché et la peur: la culpabilisation en Occident XIII'-XVIII' siècles, Paris, Fayard, 1983, p. 365 et passim.

(2) L'Amphitheatre sanglant où sont representees plusieurs actions tragiques de nostre temps, Paris, J. Cottereau, 1630; Les Spectacles d'horreur où se descouvrent plusieurs Tragiques effets de nostre siecle, Paris, A. Soubron, 1630; Les Rencontres funestes, ou Fortunes infortunees de nostre temps, Paris, G. Alliot, 1644. Pour les deux premiers de ces titres nous nous référerons au cours de cette étude aux éditions de S. Ferrari: L'Amphithéâtre sanglant, Paris, Champion, 2001; Les Spectacles d'horreur, Paris, Classiques Garnier, 2013.

(3) L'Amphithéâtre sanglant, «L'auteur au lecteur», p. 179: «En cela je marche après les pas de François de Belleforest et de François de Rosset, qui ont auparavant moi écrit des Histoires tragiques avec un succès assez heureux». 
et autres faits divers). Des récits dont le développement narratif, comme l'observe Anne de Vaucher-Gravili dans une étude célèbre ${ }^{4}$, peut se ramener sommairement à un schéma ternaire faisant se succéder les temps de l'interdiction, de la transgression et du châtiment des coupables. Des récits dont l'énonciation revient à un narrateur s'attribuant le rôle de juge et de moraliste. Ainsi leur texte est régulièrement précédé d'un argument alertant le lecteur sur le contenu moral, et il se termine tout aussi souvent par un message édifiant. Enfin, des récits exemplaires, eu égard à la solidarité de l'exemple et du contre-exemple dont la fonction est de mettre en gardes.

Tout cela a déjà été dit et bien dit $^{6}$, mais le profil spécifique de l'histoire tragique à la manière de Camus, s'il a fait l'objet de plusieurs approches éclairantes ${ }^{7}$, gagnerait encore, nous semble-t-il, à être précisé à la lumière de sa pensée anthropologique ${ }^{8}$ telle qu'elle s'exprime dans son œuvre proprement religieuse. Cette étude conduira précisément à progresser sur ce chemin, avec la conviction - ce sera là son fil directeur - que sa conception des passions telle qu'elle est formulée dans plusieurs de ses textes - principalement le Traitté des passions de l'âme et le Traité de la réformation intérieure $^{10}$ - a profondément agi sur son travail d'écrivain.

(4) A. de Vaucher-Gravili, Loi et transgression. Les histoires tragiques au XVII siècle, Lecce, Milella, 1982.

(5) Sur les liens de l'histoire tragique, de l'exemplarité et du contre-exemple voir T. Catel, Le sentier de l'exemple: morale et moralisation dans la nouvelle tragique en France de 1559 à 1630. Thèse soutenue à l'Université Paris IV-Sorbonne, le 9 décembre 2016.

(6) On se contentera de donner ici quelques repères dans une bibliographie très abondante: A.-M. Schmidt, Histoires tragiques, in Études sur le XVI siècle, Paris, A. Michel, 1967, pp. 247-259; M. Lever: De l'information à la nouvelle: les "canards" et les "bistoires tragiques" de François de Rosset, "RHLF", juillet-août 1979, pp. 577-593; S. Poli, Storia di Storie. Considerazioni sull'evoluzione della storia tragica in Francia dalla fine delle guerre civili alla morte di Luigi XIII, Abano Terme, Piovan, 1985; rééd.: Histoire d'bistoires. Considérations sur l'bistoire tragique du premier XVII siècle, éd. et trad. Ch. Rolla, Paris, Classiques Garnier, 2016 et Histoire(s) tragique(s). Anthologie/Typologie d'un genre littéraire, Fasano-Paris, Schena- Nizet, 1991; L. Sozzi, L'bistoire tragique” nella seconda metà del Cinquecento francese, Torino, Genesi, 1991; T. Pech, Conter le crime. Droit et littérature sous la Contre-Réforme: les histoires tragiques (1559-1644), Paris, Honoré Champion, 2000; J.-Cl. Arnould, L'impasse morale des histoires tragiques au XVI siècle, "RH" 57, 2003, pp. 93-108.

(7) R. Favret, Jean-Pierre Camus. Trente nouvelles, Introduction, Paris, Vrin, 1977, pp. 1-45; E. Boggio Quallio, La structure de l'bistoire tragique de Jacques Yver à Jean-Pierre Camus, in L'Automne de la Renaissance, éd. J. Lafond et A. Stegmann, Paris, Vrin, 1981, pp. 209-218; S. Poli, Autour de Rosset et Camus: l'bistoire tragique ou le bonbeur impossible, "Littératures classiques" 15, octobre 1991, pp. 29-39; S. Robic-de Baecque, Le Salut par l'excès. Jean-Pierre Camus (1584-1652), la poétique d'un évêque romancier, Paris, Champion, 1999; J. Zufferey, Fiction et vérité dans les nouvelles de J.-P. Camus, "Poétique" 124, 2000, pp. 475- 484; S. Ferrari, Histoire tragique et grande histoire, in Littérature et bistoire au XVII siècle, "Dalhousie French Studies" 65, décembre 2003, pp. 18-36 et L'bistoire tragique au service de la cause tridentine. Exemplarité et foi religieuse dans L'Amphithéâtre sanglant et Les Spectacles d'horreur de Jean-Pierre Camus in Roman et religion de Jean-Pierre Camus à Fénelon, dir. Fr. Greiner, "Littératures classiques" 79, 2012, pp. 112-126; J. Reyer de la Rosa, "Les Rencontres funestes": séduction et efficacité morale du récit minimaliste, "Dix-septième siècle" 251 , 2011/2, pp. 213-220; T. Catel, Les histoires tragiques de Camus: les faits divers, nouveaux lieux communs? in Politique des lieux communs, dir. L. Forment, T. Poquet, L. Stamboul, La Licorne, PUR, 121, février 2016, pp. 137-150.

(8) Notons cependant la parution récente de l'excellent article de Y. Rodier qui aborde la question des passions dans les histoires dévotes de Camus, mais en adoptant un point de vue un peu distinct du nôtre: Les passions haineuses dans les histoires dévotes de Jean-Pierre Camus: un théâtre didactique des corruptions bumaines in Représenter la corruption en France à l'âge baroque, dir. Fr. Lestringant et A. Paschoud, "Revue Études de lettres", 3-4, 2015, pp. 118-135.

(9) Traitté des passions de l'âme, éd. M. Vernet, Paris, Classiques Garnier, 2014. Cette édition critique nous servira de texte de référence.

(10) Traité de la réformation intérieure, selon l'esprit du B. François de Sales, Paris, S. Huré, 1631. 


\section{Des histoires providentielles et passionnelles}

Une première caractéristique de ces histoires, quand on les compare avec celles des contemporains de Camus comme Laffemas, Rosset ou Malingre, tient à leur enracinement dans une conception religieuse de la Justice. L'évêque de Belley ne manque pas une occasion de souligner l'infériorité de la Justice des hommes sur la Justice divine. Il se peut, certes, que les coupables soient rattrapés par celle-là - plusieurs de ses histoires se terminent par le moment attendu de l'exécution publique du criminel - mais cela n'arrive souvent qu'avec l'aide de la Providence, seule à pouvoir faire infailliblement surgir la vérité $e^{11}$. La crainte du châtiment devrait donc surtout provenir de la conscience que rien n'échappe au regard de Dieu et qu'il exerce implacablement une justice rétributive proportionnant les peines infligées aux fautes $\operatorname{commises}^{12}$. Ce providentialisme n'est pas propre à Camus. Il est prégnant dans de nombreuses histoires morales, prodigieuses ou tragiques écrites avant lui par de nombreux auteurs, par exemple Daniel Drouin, qui dans l'épître d'un recueil d'anecdotes significativement intitulé Les Vengeances divines déclare s'être «dés long temps estudié de recueillir des saintes Escritures de divers autheurs» des exemples capables de «degouster ceux qui $[. .$.$] sont enclins» aux vices et d'«effrayer les plus incorrigibles par les redou-$ tables chastiements du Tout-Puissant ${ }^{13}$. Cette morale terrifiante appuie son efficacité sur l'évocation de punitions prodigieuses dont l'évocation persiste chez Camus. Nombre de ses histoires font intervenir, sur un mode spectaculaire, la main de Dieu dans les affaires humaines, pour mieux châtier comme il convient ceux qui échappent à la perspicacité des magistrats. Ainsi dans «Les trois têtes», une histoire figurant dans Les Spectacles d'borreur ${ }^{14}$, un brigand allemand se trouve convaincu d'homicide, grâce à la métamorphose miraculeuse de trois têtes de veaux prenant momentanément les visages de trois de ses victimes. Ailleurs, dans L'Amphithéâtre sanglant, le cadavre d'un enfant se met à saigner en présence de celle qui l'a assassinée ${ }^{15}$.

Mais il faut s'empresser d'ajouter que Camus recourt modérément à ce folklore horrifique. Sa marque propre se trouve ailleurs, dans son intérêt de psychologue et de moraliste pour la genèse des erreurs et des crimes. Il faut observer, de ce point de vue, que sa représentation de la Providence prend dans ses histoires une tournure originale: il apparaît nettement qu'elle exerce une justice en accord avec les lois psychologiques inscrites dans la nature humaine. Elle se sert en effet le plus souvent des passions pour s'accomplir, car les passions, dès lors qu'elles sont appliquées à de mauvais objectifs, entraînent ceux qui les vivent dans une logique de mort: «ces passions aveugles traînent toujours ceux qui les suivent en des précipices horribles, et les portent à des fins tragiques et misérables» ${ }^{16}$, lit-on, par exemple, à la fin d'une des histoires de L'Amphithéâtre tragique. On peut parler dans ces cas, nombreux, d'une conception immanente de la Justice providentielle. Mais il arrive aussi que la faute commise ne débouche pas directement sur son châtiment et qu'un personnage se voie injustement condamné ou puni, pour une faute dont il n'est pas l'auteur. Il faut

(11) Voir L'Amphithéâtre sanglant, p. 199: «O Dieu il n'appartient qu'à votre puissance adorable de faire sortir la lumière du milieu des ténèbres», p. 205: «la main de Dieu paraiss[ait] visiblement en cette punition», et passim.

(12) Ibidem, p. 306: «Dieu fera une importante rétribution aux insolents et aux superbes», «Vous serez mesurés de la même mesure dont vous mesurez les autres».

(13) Les Vengeances divines, Paris, J. Mettayer, 1595. Épître «Au tres-chrestien [...] Prince Henry le Grand, quatriesme du nom», f. III.

(14) Les Spectacles d'borreur cit., pp. 164-166.

(15) L'Amphithéâtre sanglant cit., p. 310.

(16) Ibidem, p. 294. 
comprendre alors que la sanction - qui peut être une erreur judiciaire - est seulement la cause occasionnelle d'un châtiment mérité, et comme déjà provoqué par les agissements passés de la victime. Camus évoque ainsi, entre autres exemples, un mauvais fils qui, après avoir battu sa mère, se trouve accusé d'un crime qu'il n'a pas commis ${ }^{17}$ ou un assassin occis à la suite d'un tragique quiproquo en raison de sa «funeste ressemblance» avec un autre homme ${ }^{18}$. Pour punir ces coupables, la Providence, dans l'un et l'autre cas, redevient une force transcendante agissant de l'extérieur sur leur destin pour faciliter la réalisation d'une fin morale. En insistant sur la puissance de cette Justice réalisée «par des moyens inconnus à la prudence des hommes» ${ }^{19}$, Camus échappe au psychologisme et à l'accusation de pélagianisme. Il n'en demeure pas moins, ici comme ailleurs, toujours attentif aux motifs psychologiques. Le recours à la Providence coordonné aux désordres de la nature humaine apparaît chez lui comme la garantie que la cause passionnelle conduira sûrement à son effet. Cette conviction retentit sur son art d'écrivain et le conduit vers un traitement particulier de l'histoire tragique qui, sous sa plume, évolue vers une forme nouvelle: celle d'un théâtre de la vie morale où les événements sont toujours préparés, doublés, dénoués par une histoire intérieure qui en éclaire le sens profond.

\section{L'exemplarité des histoires tragiques}

Puisque les passions, avec l'aide de la Providence, sont les principaux instruments de la Justice, Camus choisit de les mettre en scène dans des histoires conçues pour mettre en garde contre leur pouvoir dévastateur. Comme tous les auteurs d'histoires tragiques, il fait du récit sinistre des erreurs humaines une forme de contreexemple et joue sur le registre de l'effroyable, pour susciter la crainte du lecteur: la peur du châtiment. Chez lui, cependant, la représentation du crime n'est pas seulement une source d'émotions puissantes, elle se double d'une réflexion profonde sur la nature du mal qui agit aussi sur sa vision de l'exemplarité. Le mal tel qu'il le représente apparaît le plus souvent (sauf pour quelques délinquants invétérés définitivement enlisés dans leurs mauvaises habitudes) comme une forme d'égarement prenant ses victimes par surprise en court-circuitant leur capacité de réflexion comme dans «L'inconsidération désespérée» ${ }^{20}$, au titre particulièrement révélateur. Dans cette histoire des Spectacles d'Horreur un laboureur impatienté par l'un de ses enfants lance une pierre dans sa direction et l'atteint «plus malheureusement que violemment en tel endroit de la tête qu'il le couche raide mort sur le champ ${ }^{21}$. De désespoir, il se suicide. La suite va dans le sens de l'amplification du malheur initial:

Quelque voisin, découvrant cet horrible spectacle, court incontinent le dire à sa femme, qui nouvellement relevée de couche remuait son petit enfant devant son feu. Effrayée de cette nouvelle, elle y court, laissant par inadvertance tomber son enfant dedans le feu, qui y fut incontinent suffoqué. Revenue de sa grange, où elle avait vu son mari étouffé, elle trouva son enfant mort et brûlé à demi, de quoi saisie de rage, sans faire autre bruit, elle s'alla pendre auprès de son mari ${ }^{22}$.

(17) Les Rencontres funestes cit., p. 175.

(18) Les Spectacles d'borreur cit., p. 97.

(19) Les Rencontres funestes cit., p. 175.

(20) Les Spectacles d'borreur cit., pp. 76-78.

(21) Ibidem, p. 77.

(22) Ibidem. 
Ainsi le malheur procède souvent d'un «débile principe [...] d'où vous apprendrez», écrit Camus, que le diable «n'est pas sans raison dans les saintes pages appelé fourmi-Lion, pour ce qu'au commencement il paraît petit comme une fourmi, mais à la fin il se montre un Lion dévorant» ${ }^{23}$.

Mais se défier des passions ne doit pas revenir à les rejeter. Sur ce point Camus s'écarte des positions stö̈ciennes. Son dessein n'est pas d'inciter le lecteur à se couper de ses sentiments sous prétexte qu'ils peuvent l'entraîner sur une pente dangereuse, mais de l'inviter à mieux comprendre leur logique afin d'apprendre à mieux les régler. La passion, en effet, de son point de vue qui doit beaucoup à Thomas d'Aquin ${ }^{24}$, est une énergie indifférente en soi, tirant sa valeur positive ou négative du but où elle est appliquée. Cette position mesurée permet de comprendre que le contre-exemple ne fonctionne jamais dans son œuvre comme la représentation d'un mal absolu. Il est toujours explicitement donné comme l'image d'un mauvais emploi des passions par manque de lucidité, faute de raison.

Loin d'être toujours traité comme un motif de terreur, ce contre-exemple sert aussi de point de départ à une pédagogie salutaire: «Tu trouveras parmi ces épines beaucoup de roses, et même des fruits d'énorme grosseur, et d'une douceur singulière», écrit Camus dans la préface des Spectacles d'borreur où il se compare aussi à un bon Chirurgien qui guérit «en maniant les plaies des blessés, et en tirant le sang des veines des malades» ${ }^{25}$. Au début de L'Amphithéâtre sanglant il parle de «la Morale, roulant sur ces deux pôles, la fuite de ce qui est mauvais, la suite de ce qui est bon» ${ }^{26}$. L'originalité de ses trois recueils tient justement en ce qu'ils accordent aussi beaucoup à cette poursuite du bien qui marque - de manière étonnante dans ce genre littéraire - la physionomie de quelques unes de leurs histoires. Certaines d'entre elles, en effet, loin de s'achever par la punition attendue du coupable, font évoluer la chronique criminelle vers une fin heureuse: ce sont parfois des mariages ${ }^{27}$, parfois des rédemptions de pécheurs enfin repentis et touchés par la grâce ${ }^{28}$ et assez souvent des retraites derrière les murs d'un couvent ou d'un monastère ${ }^{29}$. On le voit à ces quelques observations, du fait de sa représentation dynamique de la vie passionnelle, Camus brouille la relation d'opposition faisant du contre-exemple l'exact contraire d'un modèle moral. Il y a entre les deux, nous suggère-t-il, une nécessaire continuité, mieux encore, l'un préfigurerait l'autre et formerait comme son ébauche maladroite. «Les pires actions», dit-il en filant une métaphore médicale, font «les plus salutaires exemples, puis que le venim mesme entre dans la composition de la theriaque» ${ }^{30}$. Les titres de certaines de ses histoires reflètent clairement cette utilité paradoxale du mal comme «Le désespoir honorable» ${ }^{31}$, «L'infortune fortunée» ${ }^{32}$ ou «l'heureuse blessure ${ }^{33}$. Dès lors, rejoindre le modèle positif ne pourrait se faire simplement par le rejet d'un mauvais exemple. Puisque le mauvais exemple ne saurait être rejeté, pas plus qu'il ne saurait être imité, il devient pour le lecteur une forme de provocation

(23) Ibidem, p. 76.

(24) Voir Somme théologique, première section de la deuxième partie, question 24, Paris, Éd. du Cerf, 1994, pp. 182-186. Cf. J.-P. Camus, Traitté des passions de l'ame, ch. IV, pp. 80-90.

(25) Les Spectacles d'horreur cit., p. 65.

(26) L'Amphithéâtre sanglant cit., p. 180.

(27) Les Rencontres funestes cit., p. 74 et 120.

(28) Ibidem, p. 162.

(29) L'Amphithéâtre sanglant cit., pp. 192-199, Les Rencontres funestes cit., p. 85, 93, 98, 109.

(30) «Parole au Lecteur» in Les Rencontres funestes: préface non paginée.

(31) Les Spectacles d'borreur cit., pp. 183-185.

(32) Les Rencontres funestes cit., pp. 116-120.

(33) Ibidem, pp. 162-167. 
intellectuelle. Il n'est pas radicalement mauvais, il manifeste de manière spectaculaire un dysfonctionnement, il résulte du mauvais emploi d'une énergie pourtant utile. Il pose a contrario la question de cette utilité. Il est ainsi invitation à l'introspection, à l'analyse et au discernement seuls capables de corriger le penchant naturel des passions vers leur dérèglement.

\section{L'art du conteur}

L'exemple pour Camus se conjugue avec un art de raconter qui va à l'essentiel sans jamais se perdre dans les détails, cela a souvent été observé, à commencer par lui-même. Ainsi ses histoires tragiques se distinguent par leur grande sécheresse. L'auteur ne cesse en effet de multiplier visiblement les ellipses en les justifiant. Il ne reproduira pas telle lettre galante, il ne se fera pas l'écho de tel entretien sentimental, il ne peindra pas la genèse d'une passion amoureuse afin de bannir de ses récits toute complaisance ${ }^{34}$. Dans la préface des Rencontres funestes, il fait d'ailleurs la liste de tous les ajouts que devrait faire à sa pièce un poète dramatique qui aurait l'idée de transformer l'une ou l'autre de ses histoires en tragédie. Il lui faudrait donner plus de consistance aux personnages, ajouter des descriptions, des «pourparlers», des digressions... Au lieu de s'attarder sur les multiples actions et circonstances qui sous la plume d'un Rosset se transforment régulièrement en scènes romanesques, Camus évoque en passant et parfois, pour mieux motiver sa répugnance à dire précisément les choses, lance une pique contre l'héroïsme guerrier des duellistes ou le sentimentalisme béat des amants passionnés, formant pour lui autant de pièges tendus à l'amour propre du lecteur. L'histoire déroulée au pas de course comporte quelques pauses spectaculaires où prend place le tableau de la mort violente saisissant brusquement les criminels et leurs victimes. Mais ce spectacle d'horreur loin d'être au centre d'une scénographie visant le déploiement d'une description suggestive est relié à une action rapidement développée, moins faite pour satisfaire la curiosité malsaine par un luxe de détails horribles que pour conduire la tension dramatique inhérente à l'action vers le moment de son acmé. Dès lors, autant que sur l'horreur inhérente au spectacle, c'est sur l'enchaînement des faits qu'est attirée notre attention, comme dans ce drame de la jalousie où une jeune femme assassine son amant à coups de poignard durant son sommeil:

Pour dire les furies qui agitaient la désespérée Lucrèce, il faudrait n'être pas lié aux bornes de la brièveté d'une simple narration. Elle veut mourir, mais ce sera après avoir assouvi sa vengeance. Comme elle était dans ce furieux trouble d'esprit, Paulin la va voir, lui raconte que malgré tous les efforts de son père, il lui a conservé sa fidélité; elle prend tout cela pour des fables, plus il jure, moins elle le croit. Que ne lui dit-elle? Il la veut apaiser par caresses, mais elle le repousse, vomissant contre lui tout ce que la forcènerie peut tirer d'une bouche dont la rage possède / le cœur. À la fin étant aucunement déchargée du venin qu'elle avait en l'âme pour acheminer son sanglant dessein, elle feint de prêter quelque créance aux serments de celui qu'elle tient pour le plus perfide qui soit au monde, et s'apprivoisant, elle lui permet de prendre part en son lit comme son Époux légitime. Aussitôt qu'elle le sentit endormi (ô aveuglement brutal d'une jalousie désespérée!), elle lui enfonce par plusieurs fois un grand couteau qu'elle avait préparé pour cette horrible exécution dans la gorge, dans l'estomac, dans le ventre, et à coups redoublés elle chasse l'âme du corps du déplorable et trop loyal Paulin. Et puis, après avoir assouvi ses yeux sanguinaires d'un Spectacle si tragique, et comme bu ce sang

(34) Voir par exemple L'Amphithéâtre sanglant cit., p. 280; Les Spectacles d'horreur cit., p. 160. 
qui demandait vengeance contre celle qui le répandait avec tant d'injustice, elle tourna comme une Ourse blessée, et qui a la flèche dans le flanc, sa rage contre soi-même, et avec le même fer qu'elle enfonça dans son estomac, elle se fit sentir la même cruauté qu'elle venait de faire expérimenter au misérable Paulin ${ }^{35}$.

La composition de cette séquence, qu'il est facile de diviser suivant le plan d'une progression en trois étapes, glisse du temps de la motivation («les furies qui agitaient la désespérée Lucrèce») et des circonstances («Paulin la va voir»), vers ceux de l'assassinat, puis du suicide. Entre le deuxième et le dernier temps, il est vrai que Camus introduit une légère pause, un arrêt sur l'image du cadavre de Paulin reliée au point de vue de Lucrèce («[... Et puis, après avoir assouvi ses yeux sanguinaires d'un Spectacle si tragique $[. ..] \gg)$. Mais cette image nous est, pour ainsi dire, rendue de manière oblique, à travers les réactions qu'elle engendre chez l'assassin. On devine la raison de ce décalage: Camus veut moins coller à la vision qu'à ses effets délétères et au mécanisme fatalement autodestructeur menant de la jalousie vers le crime, puis du crime vers le suicide. Très intéressante de sa manière particulière de représenter l'horrible est le commentaire qui suit immédiatement «cette funeste aventure»:

Représentez-vous s'il vous plaît ce Spectacle d'horreur, et y considérez toutes les particularités. Voilà deux morts entassées l'une sur l'autre à raison d'un faux bruit et d'une mésintelligence. Paulin meurt pour être trop fidèle à cette barbare qui, non moins impitoyable à ellemême qu'à son trop loyal Amant, perd par une jalousie précipitée, et son corps et son âme ${ }^{36}$.

Les «particularités» de cette représentation se trouvent moins dans l'image concrète des «deux morts» que dans l'enchaînement des gestes et des sentiments ayant conduit Paulin et Lucrèce vers leur fin sinistre. Le théâtre d'horreur de Camus nous livre ainsi son sens moral: ses principaux acteurs sont les passions présentées comme aussi redoutables que les effets sanglants dont elles seraient les causes.

Ces quelques analyses ponctuelles pourraient être étendues à l'ensemble des histoires des trois recueils. Ici et là, en effet, le clou du spectacle ce sont bien les passions et leurs ravages, comme le signale explicitement Camus attirant constamment notre attention sur les enjeux moraux de ses histoires: «Vous allez voir une iniquité prolongée par l'espace de dix-huit ans avec une inhumanité qui vous donnera sans doute de l'horreur si vous n'êtes pas dépouillé de tout sentiment ${ }^{37}$; «vous allez voir un grand exemple de jalousie et un spectacle d'horreur sur une punition effroyable» ${ }^{38}$; «apres l'heureux trepas d'un Libertin, nous ferons suivre la deplorable mort d'un autre, non qu'elle ait rien de sanglant pour le corps, mais elle est bien redoutable pour l'âme» ${ }^{39}$.

L'emploi constant d'un style elliptique associé à un tempo rapide permet de livrer au lecteur des scénarios à peine étoffés et ramenés à leurs rouages les plus élémentaires: les passions, toujours comprises comme la cause des actions et scrutées dans leurs effets ${ }^{40}$. Ces passions sont suivies et examinées pas à pas à partir du moment de leur genèse. L'origine du mal est souvent insignifiante. Ainsi, nous l'avons vu à propos de «L'inconsidération désespérée», «une petite étincelle» est à l'origine d' «un grand

(35) Les Spectacles d'borreur cit., pp. 174-175.

(36) Ibidem, p. 175.

(37) Ibidem, p. 107.

(38) Ibidem, p. 115.

(39) Les Rencontres funestes cit., p. 168.

(40) Les Spectacles d'borreur cit., pp. 103-104: «Notre histoire raconte bien au long et / la cause et les effets de son mécontentement», écrit Camus à propos du protagoniste du «Serment méprisé». 
embrasement ${ }^{41}$. Après avoir pointé une faiblesse initiale, Camus fait voir ses «progrès» en montrant comment une première faute en amène d'autres plus graves par le biais de passions toujours plus puissantes. Sa narration suit fréquemment à cet égard la ligne d'un crescendo dramatique puisant dans les procédés rhétoriques de l'hyperbole, de l'amplification, de l'accumulation ou de «l'entassement» pour reprendre l'une de ses expressions favorites ${ }^{42}$. Cela l'amène souvent à enchaîner rapidement les catastrophes dans le cadre d'une même histoire. Dans «Les morts entassées» ${ }^{43}$ le suicide d'une veuve infanticide provoque la mort de son amant, puis du prêtre qui avait refusé le baptême à son enfant; dans «les Duels entassez» ${ }^{44}$ la beauté d'une jeune femme provoque autour d'elle de nombreux affrontements mortels entre ses soupirants. À la fin de «L'injuste jalousie», Camus évoque le «funeste effet» de cette passion, «qui entasse tant de morts pour son seul ombrage» ${ }^{45}$. Les histoires les plus simples, où les catastrophes ne se déclinent pas en séries, obéissent néanmoins à une même logique du comble, intéressant autant l'évolution calamiteuse des sentiments que celle des faits, et conduisant par paliers successifs vers le moment d'une crise spectaculaire rapidement dénouée, le plus souvent par la mort du coupable.

\section{Le cadre rhétorique et le didactisme}

La voix du conteur est constamment doublée dans les histoires tragiques par celle du commentateur qui prend position face à l'action représentée, parfois s'indigne et interpelle ses personnages, apostrophe le lecteur. On retrouve chez Camus le même dispositif énonciatif, avec cette différence cependant que chez lui s'affirme plus nettement qu'ailleurs l'influence d'un intertexte religieux sur l'écriture du nouvelliste. En écrivant ses histoires, Camus semble constamment se souvenir de ses homélies. Il emprunte non seulement à leur matière et à leur cadre formel, mais il s'attache aussi, tout en racontant, à amener le lecteur, comme le prêtre s'adressant à son auditoire, vers une meilleure compréhension de sa vie morale.

Les titres de ses histoires, comme ceux de ses sermons, placent souvent l'accent sur un sentiment, une passion, une inclination morale, avec cette différence cependant que les premières les abordent seulement par un angle négatif. Les histoires de L'Amphithéâtre sanglant se présentent ainsi, entre autres exemples, sous les intitulés de «L'avare infortuné», «L'infidèle châtié», «La sanglante chasteté», «Les injustes parents» alors que l'on trouve dans les pages des Homélies quadragésimales (Rouen, J. Osmont, 1625) des titres comme «Du mespris de la Patrie», «Du Jugement temeraire», «La Pecheresse penitente» ou «De la mort d'Amour».

Les exordes sont souvent semblables. L'orateur chrétien commence ses homélies par l'annonce d'un thème appuyé par l'autorité de l'Écriture Sainte, ou par une observation tirée de l'historiographie profane ou de la philosophie naturelle, et à la lumière de ces premières réflexions sont exposées les règles de la vie chrétienne. Camus auteur d'histoire tragique débute souvent de la même manière en utilisant parfois exactement les mêmes formules. On devine à cet égard qu'il possède des cahiers de lieux communs dans lesquels il puise abondamment. Reportons-nous par

(41) Ibidem, p. 76.

(42) Employée, par exemple, au début des Spectacles d'borreur, p. 69: «Je ferai l'ouverture de ces spectacles d'horreur par un entassement de meurtres».

(43) Ibidem, pp. 180-182.

(44) Les Rencontres funestes cit., pp. 182-188.

(45) Ibidem, p. 69. 
exemple à l'introduction de «l'Amant sacrilège» évoquant la «punition effroyable, arrivée à un gentilhomme espagnol» ayant tenté de séduire une nonne ${ }^{46}$. L'écrivain, sur le modèle de l'orateur sacré, y multiplie les références bibliques au livre de l'Exode (20, 5: «Je suis le Dieu fort et jaloux»), au premier livre des Rois où Dieu châtie les Philistins qui se sont emparés de l'Arche d'alliance, au chapitre V du livre de Daniel où Balthazar dans un acte sacrilège boit à ses Dieux dans des vases dérobés au temple de Jérusalem, à un verset du livre d'Isaïe $(28,20$ : «Le lit sera trop court pour qu'on s'y étende, / La couverture trop étroite pour qu'on s'en couvre». Il ajoute à cela une image empruntée à un bestiaire qu'il a déjà utilisée sous une formulation voisine dans ses Homelies panegyriques de S. Ignace de Loyola ${ }^{47}:$ «n dit que le Lion trouvant le Léopard auprès de la Lionne entre en une si grande fureur qu'il met en pièces ce rival» ${ }^{48}$. L'histoire, qui commence immédiatement après ce paragraphe, prend son sens plein d'être lue à partir d'un tel intertexte: c'est en effet grâce à celui-ci qu'il fonctionne véritablement comme un exemple, c'est-à-dire comme un cas, certes singulier, mais actualisant et validant aussi une vérité première d'abord inscrite dans la Bible ou le livre de la création auquel renvoient les mœurs léonines.

Le corps de l'histoire tragique, autrement dit son développement narratif, se distingue évidemment de celui de l'homélie divisée en différents points ordonnés suivant un fil logique. On peut même dire qu'il obéit à un ordre de présentation inverse puisqu'il va du particulier vers le général alors que le discours religieux illustre ses vérités générales par des exemples particuliers. Persiste cependant entre les deux un dénominateur commun, celui de l'exemplification (par déduction ou induction) qui établit une relation entre l'anecdote et son application possible à la vie de chacun, le cas singulier et une vérité morale universelle. Dans les trois recueils ce lien est constamment tissé entre l'histoire et la leçon à laquelle elle sert de support. Les personnages font l'objet d'une présentation généralisante parfois portée par l'onomastique (Artaban pour un personnage orgueilleux ${ }^{49}$, Gorgonia pour une mégère assoiffée de sang ${ }^{50}$, Formose pour un séducteur $\left.{ }^{51}\right)$. Ils peuvent être donnés pour des représentants typiques de leur nationalité, de leur position sociale, de leur métier régulièrement associés à une caractérologie ${ }^{52}$, ou comparés à des figures historiques, mythologiques ou bibliques $^{53}$ voire à des animaux ${ }^{54}$. L'action et les événements sont éclairés par des citations bibliques, des proverbes, des maximes définissant ensemble les règles d'un code moral transcendant les lieux et les époques ${ }^{55}$. Toujours attentif à la logique passionnelle sous-tendant ses histoires, Camus use aussi volontiers de l'allégorie qui, sous sa plume, évoque parfois le genre ancien de la psychomachie. Ainsi les drames qu'il dépeint trouvent-il à se prolonger dans un décor symbolique illustrant les luttes et les débats intérieurs. Afin de décrire les manœuvres de l'un de ses person-

(46) Les Spectacles d'borreur cit., p. 115

(47) Homelies panegyriques de S. Ignace de Loyola, Lyon, J. Gaudion, 1623. Cf. p. 345: «Ignores-tu que le Lyon recognoissant en sa lionne quelque sentiment de l'accointance du Leopard la met en mille pieces pour contenter la rage de sa jalousie?».

(48) Les spectacles d'borreur cit., p. 115.

(49) Ibidem, p. 252.

(50) Ibidem, p. 296.

(51) Ibidem, p. 187.

(52) Ibidem, p. 317: sur la jalousie des Italiens; Les Spectacles d'borreur, p. 103, sur «les serments des soldats [...] aussi peu solides que ceux des amoureux» et passim.

(53) Les Spectacles d'borreur cit., p. 125: «au lieu de se repentir comme David»; p. 143: exemple d'un sénateur romain...

(54) Les Rencontres funestes cit., p. 268: avec un chien et un pourceau...

(55) Voir par exemple L'Amphithéatre sanglant, p. 188: «Les Troyens, dit l'ancien proverbe, se repentent mais trop tard»; ibidem, p. 196: «la chair bien repeuë se revolte aisement contre la raison», et passim. 
nages venu à la Cour pour y quêter une vaine gloire, il emploie par exemple les images du vent, de la mer et du nautonier déjà utilisées dans son Traitté des passions de l'âme ${ }^{56}$ pour décrire leurs effets sur l'humanité:

Tryphon trouva tant de difficultés à surmonter, et tant d'obstacles à son dessein, qu'encore qu'il fût excellent courtisan, il y perdit toute sa finesse, semblable à ces bons nautoniers qui dans une furieuse tourmente perdent leur science, la violence de la tempête étant plus forte pour attaquer leur vaisseau que leur art n'est subtil pour se défendre des vagues ${ }^{57}$.

Plus loin, d'une veuve qui songe à se remarier avec un tout jeune homme, il écrit que «le remords de l'intérêt l'empêcha de cingler à pleines voiles où elle désirait» ${ }^{58}$.

Enfin - point essentiel dans l'utilisation du contre-exemple - les commentaires du narrateur s'inscrivent constamment en contrepoint des paroles et des points de vue de ses personnages qui ne sauraient, évidemment, être pris sans distance. À cet égard, Camus ne manque jamais de souligner que les protagonistes de ses histoires sont coupés d'une juste perception du réel. Pour mettre leur folie en évidence il utilise régulièrement les mêmes images évoquant la cécité ou une vision altérée par les illusions: «Il en est de la colère et de l'amour - écrit-il ainsi dans «L'intempérance précipitée» - comme des chiennes: celles-ci produisent leurs petits aveugles et ces passions ne font leurs actes qu'avec aveuglement» ${ }^{59}$. Dans la même histoire, il parle, en des termes tout aussi imagés «des fausses lunettes de cette lunatique passion qui s'appelle jalousie» ${ }^{60}$.

La conclusion des histoires condense leurs leçons dans une ou plusieurs formules frappantes. Parfois la péroraison est réduite au minimum, comme pour laisser le lecteur sur une forte impression. Une seule phrase alors suffit à dégager le sens du dénouement violent. Plus souvent, la fin est nettement délimitée par une incitation explicite à tirer la morale de l'histoire ${ }^{61}$, souvent suivie par divers propos édifiants: recommandations, prières, citations bibliques, phrases à méditer que l'on trouve aussi dans les sermons et les homélies. Mais Camus use aussi d'un procédé qui, s'il provient encore des discours religieux, est particulièrement adapté aux histoires: il s'agit de la récapitulation ${ }^{62}$ reprenant de manière synthétique les différents points abordés au cours de la narration. La récapitulation permet au conteur de dégager les lignes de force sous-tendant son récit et de le réduire à son squelette moral pour mieux l'imprimer dans la mémoire du lecteur:

Que de remarques nous présente cette Histoire! - écrit-il par exemple pour conclure une des histoires de L'Amphithéâtre sanglant - La prééminence de l'amour sur l'amitié, la perfidie d'un ami, la stupidité d'un mari, l'infortune d'une honnête femme, la punition humaine et divine d'un ravisseur qui, échappant les mains de la Justice de la terre, tomba en celle du Ciel, et enfin le bonheur de Ménodore, qui par ces tempêtes fut poussé au port heureux de la Religion, et sa générosité à mépriser un bien qu'elle ne pouvait posséder sans horreur, encore qu'il lui fût acquis légitimement par la voie de la Justice ${ }^{63}$.

(56) Cf. Traitté des passions de l'âme cit., ch. VIII, p. 119.

(57) L'Amphithéâtre sanglant cit., p. 281.

(58) Ibidem, p. 351.

(59) L'Amphithéâtre sanglant cit., p. 311.

(60) Ibidem, p. 312.

(61) Voir L'Amphithéâtre sanglant cit., p. 237; Les Spectacles d'borreur cit., p. 147; Les Rencontres funestes cit., p. 29.

(62) Direction à l'oraison mentale, Lyon, P. Rigaud, 1623: «J'entends par la recapitulation une reveüe speciale de l'oraison, et une reflexion sur toutes ses parties pour en recueillir les fruicts».

(63) L'Amphithéâtre sanglant cit., p. 366. Voir aussi ibidem p. 294 et Les Spectacles d'borreur cit., p. 273. 
Ainsi résumée en quelques mots, l'histoire achève de se dépouiller de tous ses ornements inutiles pour devenir, conformément au vœu de l'écrivain, un exemple offert à la méditation du lecteur.

\section{La visée morale}

Il ne sera pas inutile, enfin, de se demander comment Camus se représente la visée morale de ses histoires. Certes, il s'agit pour lui de mettre en garde les lecteurs, en suscitant peur et répulsion, et de les inciter aussi à réfléchir sur les racines passionnelles de la méchanceté, mais encore faut-il comprendre précisément comment l'usage des exemples édifiants fournis par les histoires tragiques se relient dans son œuvre à une pédagogie servant le salut chrétien.

Dans son Traitté des passions de l'âme, il revient sur la signification habituellement prêtée à la crainte: «On la peut deffinir ainsi; - écrit-il en se fondant sur Aristote et saint Augustin ${ }^{64}$ - Une apprehension ou fuitte du mal futur, ou d'un peril imminent»s. Mais comment d'une inclination négative pouvant être assimilée à un manque de courage pourrait naitre un mouvement utile sur le plan moral? Par la loi psychologique de ce qu'il nomme «l'application». Il faut rappeler ici que les passions ne sont pas pour lui mauvaises en soi, mais qu'elles constituent des puissances neutres, bonnes ou néfastes suivant l'utilisation qui en est faite. Il suffirait donc dans cette logique d'appliquer la crainte à un but estimable pour en faire le levier d'un progrès vers la vertu chrétienne. Ainsi Camus se réfère à saint Paul qui nous conseille d'operer nostre salut avec crainte et tremblement en interprétant ses paroles à la lumière de sa théorie. La crainte et le tremblement deviennent pour lui une forme du souci que porterait le chrétien à son salut en les appliquant aux images anticipées du Jugement de Dieu et de l'Enfer. La crainte bien employée serait un aiguillon pour la vertu de celui qui voudrait échapper «aux supplices qui attendent les pervers en l'autre vie» et un moyen pour «les Sages» d'aller «au devant de ce pas [la mort] par la premeditation, et par une anticipation de pensée, pour n'estre surpris au depourveu, et sans preparation de ceste meutriere» ${ }^{65}$. La peur du châtiment divin accompagne de fait, on l'a vu, le développement des histoires tragiques, par leur rappel constant du rôle punitif de la Providence veillant au triomphe inévitable de la Justice. Là sans doute réside une grande partie du message moral de Camus, comme il le note clairement dans la préface de ses Rencontres funestes:

Icy donc, Lecteur, tu apprendras à detester les Gens de sang et de tromperie, que Dieu mesme a en abomination, et dont il menace de couper la vie par le milieu de son cours. Comme aussi à tenir en bride ces furieuses Passions, qui, comme des torrens impetueux, font tant de ravages en la société des hommes ${ }^{66}$.

On aurait tort cependant de ramener ses histoires à une leçon aussi sommaire. Leur logique profonde - nos précédentes analyses l'auront montré - n'illustre pas seulement cette «pastorale de la peur» par laquelle l'historien Jean Delumeau désignait un militantisme religieux insistant sur les aspects les plus inquiétants du chris-

(64) Traitté des passions cit., p. 472. M. Vernet indique les références suivantes dans son édition critique: Aristote, Rhétorique, 2, ch. 5; Augustin, Quatre-vingt-trois questions, question 33, «de la crainte».

(65) Ibidem, p. 474.

(66) «Parole au Lecteur», in Rencontres funestes. 
tianisme (notamment la peur de l'enfer) ${ }^{67}$. L'anthropologie de Camus est en effet comme couronnée par la raison, présentée par lui comme «Régente des Passions», une faculté capable de redresser «le bois tortu de nos mauvaises inclinations» et de diriger «les passions en la voye royalle de la vertu» ${ }^{68}$. Mais comment s'exerce exactement ce rôle directeur? La raison selon Camus - une raison évidemment soutenue par la foi et la religion - exerce son magistère par la conscience ou la présence à soi. Il est intéressant de voir à cet égard que pour la définir, il la présente, à partir d'une citation du Cantique des Cantiques, comme une faculté de réflexion:

Si vous ignorez, c'est à dire, si vous ne vous cognoissez raisonnable, dit l'Espoux sacré à l'ame, allez vous en paistre apres les bestes irraisonnables. C'est le plus grand traict de raison de recognoistre sa raison: Et c'est estre vrayement Roy de soy, que de donner l'Empire et maistrise à ceste Royne sur toutes ses actions ${ }^{69}$.

La raison se manifeste d'abord comme un mouvement de reconnaissance de la raison par elle-même, un retour à soi permettant au pasteur de la bible de conserver la maîtrise de son troupeau, «les bestes irraisonnables» des passions inclinant vers le mal quand elles échappent au frein de la lucidité. Cette reprise préconisée de soi par soi qui conforterait l'humanité dans son essence rationnelle et la ramènerait vers la foi permet de mieux comprendre a contrario ce que représente le mal du point de vue de Camus. Suivant sa théorie classique, le mal se définit négativement pour lui comme «privation du bien» ${ }^{70}$. La perversité est une forme d'égarement, d'aveuglement par lequel on s'absente de soi.

Revenons, pour conclure, à la question du bien moral distillé par les histoires tragiques de Camus. Les spectacles d'horreur qu'elles représentent ne doivent pas être seulement de son point de vue des motifs de peur: à travers même la peinture des débordements passionnels, toutes fonctionnent comme des incitations à rompre avec l'irréflexion pour revenir à soi, par la conscience et la vigilance. Il y a, à cet égard, dans l'Avant-propos du Traité de la Réformation intérieure une belle image où l'on peut voir comme une clé de lecture pour ses fictions exemplaires: celle d'une «ruche de verre» dont la transparence permettrait de découvrir «l'œconomie des abeilles» ${ }^{71}$. L'image des abeilles représente les facultés de l'âme, «la ruche de verre» est le livre qui permet de leur donner une visibilité et de conduire ainsi le lecteur sur le chemin du progrès intérieur d'une «Réformation» stimulée par la connaissance:

C'est à cette clarté - écrit Camus - que je me suis le plus estudié en ce petit ouvrage, où j'examine toutes les parties de nostre ame l'une apres l'autre, et apres avoir remarqué leurs deffauts, j'y applique les remedes que j'apprends des meilleurs maistres de la vie spirituelle. Je vay chercher le mal en sa source pour la tarir, et en sa racine pour y mettre la cognée. Si tu as le courage, Lecteur devot, de t'exercer en prattique de cette Reformation, je me promets avecque l'assistance de la divine grace, que tu feras un bien plus notable avancement spirituel que par celle de beaucoup d'exercices exterieurs qui cassent bien des corps, ruinent beaucoup de santez, et n'estropient que trop de cervelles ${ }^{72}$.

(67) Voir J. Delumeau, Le Péché et la peur cit., pp. 416-446.

(68) Ibidem, p. 487

(69) Ibidem, p. 485.

(70) Saint Augustin, De quaest. 83, q. 21. Cité par J.-Y. Lacoste, in Dictionnaire critique de théologie, Paris, Puf, 1998, p. 831.

(71) Traité de la réformation intérieure, Avant propos, non paginé.

(72) Ibidem. 
Le lecteur dévot visé à travers ces déclarations ne saurait représenter le plus vaste public, moins scrupuleux, des histoires tragiques. Il n'en demeure pas moins que le livre et la relation de lecture sont donnés ici et là comme les moyens d'une rédemption à réaliser avec les yeux de la raison.

FRANK GREINER

Université de Lille 